\title{
RELATIONSHIP OF DEPRESSION, AGING AND IMMUNE SYSTEM DURING COVID-19 PANDEMIC: A REVIEW
}

*Farah Iylia Binti Fauzi ${ }^{1}$, Siti Fatimah Binti Salleh², Mohammad Shahadat Hossen ${ }^{3}$

${ }^{1}$ Faculty of Language and Linguistics, University of Malaya, Kuala Lumpur, Malaysia.

${ }^{2}$ Faculty of Islamic Contemporary Studies, University of Sultan Zainal Abidin, Terengganu, Malaysia.

${ }^{3}$ Faculty of Human Ecology, University of Putra Malaysia, Selangor, Malaysia.

*Corresponding author; Email: farah.iylia@ymail.com


Received: 10 May 2021

Accepted: 20 June 2021

Published: 07 August 2021

Volume -2, Issue-3

Cite This: ICRRD Qual. Ind. Res. J. 2021, 2(3), 101-108

\begin{abstract}
COVID-19 is a highly contagious virus which first appeared in China in December 2019. It has affected over 157 million people and killed over 3.2 million. The paper reviews the function of the immune system for COVID-19 prevention, depression, and anxiety due to COVID-19 and their effects on the immune system and the relationship of aging with the immune system and depression and anxiety. It has been found that several elderly people lack the coping mechanisms required to deal with the stress caused by COVID-19. Hence, identifying the factors and mechanisms that lead to this resilience will aid in the development of preventive measures for certain elderly people and groups with more severe mental health problems. Additionally, it would be beneficial to understand how technology could be leveraged to accomplish this goal. During the pandemic, various steps such as social isolation, quarantine, and self-isolation are need to be implemented properly to slow the spread of the virus. Meanwhile, to help halt the pandemic, everybody must be vaccinated as soon as possible until any bans on social gatherings and social isolation can be removed, allowing other sectors such as schooling, social activities, and life to resume normalcy.
\end{abstract}

Keywords: COVID-19 Pandemic; Social Gatherings; Social Isolation; Mental Health; Immune System; Elderly People.

\section{INTRODUCTION}

The Covid-19 pandemic is a global issue that has infected millions and many has lost their lives to the virus. On December 31, 2019, a novel Coronavirus type (2019-nCoV) was reported as the etiological agent in cases of pneumonia of unknown origin seen in Wuhan, China (Zhu et al., 2020). The virus is new and no authorized treatment or already existed vaccine were able to battle the virus (Zhang, 2020). The nature of the disease is easily transmittable and spread from one individual to another and can easily be spread to the whole world. The virus is a 
highly contagious infectious disease, with one infected person infecting three other individuals on average (Chowdurya et al, 2020). Due to this quarantine and social distancing is one of the most crucial ways in combating the disease.

The most common disease-related symptoms were fever, cough, muscle aches and/or fatigue, dyspnea, headache, sore throat, and gastrointestinal symptoms (Nascimento et al, 2020). With the symptoms of the disease being almost similar to many influenza, many could not detect themselves if they are infected until they are tested positive. Often people do not display any signs of the infection and serve as carriers to spread this deadly infection. The main source of infection transmission is from the patient's droplets, which are inhaled into the lungs of surrounding people as he coughs, speaks, or sneezes. The virus spreads through coughing and sneezing droplets, reaches the nasal system through inhalation, and begins replicating (Chowdurya et al, 2020).

Because the virus is invisible to the human eyes, many do not recognize it whenever they are exposed to the virus or who is infected and which area is infested with the virus. These aerosols may be inhaled through the nose or mouth and enter the human body (lungs) (Chowdurya et al, 2020). To prevent the spread of COVID-19 The World Health Organization (WHO) recommends that everyone wash their hands often with soap and water after touching something, sneezing, coughing, blowing their nose, and returning from a public location. Unwashed hands should not be used to touch the face, nose, lips, or mouth as this will be dangerous in the case of touching and picking up the virus from any surfaces from public places thus washing hands could very much decrease the risk of getting infected with the virus. Wearing masks is an essential and efficient precaution for preventing the spread of coronavirus infection. It is self-evident that if people do not come into contact with one another, the risk of infection spreading is greatly reduced. Communication with people should be avoided at all costs. When going out, keep a distance of at least 1 meter between yourself and others (World Health Organisation, 2020).

In preventing the virus, $\mathrm{WHO}$ has determined that there are vaccines against COVID-19 that meet the requisite safety and efficacy requirements as of April 8, 2021. And if a person already had COVID-19, they should take whatever vaccine is made available to them first. The World Health Organization emphasises the importance of getting vaccinated as soon as possible when their turn comes around, and although no vaccine is completely safe, approved COVID19 vaccines are.

$\mathrm{nCoV}$ infection is highly heterogeneous, from asymptomatic infection to mild, moderate, or severe COVID-19. In addition, the infection can evolve through different stages and progress in either direction both in improving and recovery versus worsening and death (Guo et al, 2020).For 80 percent of infected patients, the disease would be mild and limited to the upper and conducting airways. These individuals can be treated and monitored at.

2019-home with conservative symptomatic therapy. Approximately $20 \%$ of the infected patients develop pulmonary infiltrates and some of these develop very severe disease. According to a recent epidemiological study by China CDC, the mortality rate of extreme COVID-19 patients may be as high as 49\%. (Prompetchara et al, 2020). 
By reducing the symptoms and health risks of the virus, an individual will be less likely hospitalized and this will help reduce strains on the health care facilities and reduce the potential of overwhelming the Intensive care units and the front liners. To further stop the pandemic everyone needs to get vaccinated as soon as possible before any social gatherings banned can be lifted and social distancing can be stopped so that other sectors such as education, social events, and life can return to normal.

\section{The role of the immune system in preventing Covid-19}

The immune system protects our body from infections and diseases. The organs of the immune system, which defend against diseases, are found in the body. The organs and processes that every human body has provided resistance to any infection and toxins. The organs include the thymus, bone marrow, and lymph nodes are the main organs that work to provide the body with an immune system.

However, in the case of the novel coronavirus COVID-19 which is new to the body and the immune system. There is no approved medication or vaccine against COVID-19 (Chowdurya et al, 2020). And although relying solely on the immune system is not ideal in the case of battling Covid-19, the immune system, however, is the strongest defense against COVID-19 because it promotes the body's natural ability to protect against pathogens such as viruses, bacteria, fungi, protozoa, and worms and resists infections and for COVID-19 patients', the body needs to be protected against SARS-CoV- 2 .

The availability of a vaccine against SARS-CoV-2 is fairly new and the world's population is to be vaccinated against it as there is no cure for the virus and as well as no methods to eliminate the virus from existence. So the best way to battle COVID-19 is for the body to build the immune system against it. Many are getting their dosses of the vaccines however many more are currently waiting for the vaccine while some are against being vaccinated.

\section{Effect of aging on the immune system}

There are three forms of immunity in the immune system. Innate immunity which is a rapid response, adaptive immunity which is a slow response, and passive immunity. Natural immunity, which comes from the mother since pregnancy whereas artificial immunity comes from medicine that we are prescribed against a certain disease are the two forms of passive immunity. In general, as we age our immunity changes throughout our lives, both of these branches of the immune system are affected by the aging process. Innate immunity seems to be better maintained, while the adaptive immune system undergoes more drastic and sometimes harmful age-related changes (Weiskopf et al, 2009).

As we age our body functions started to deteriorate. Aging is often linked to a reduction in the role of epithelial barriers in the skin, lungs, and gastrointestinal tract, allowing pathogenic

organisms to enter mucosal tissues and posing a greater challenge to the innate immune system of older adults (Weiskopf et al, 2009). The immune function of our body also declines with age, which leads to vaccine effectiveness being reduced in older adults (Weiskopf et al, 2009). This brings concern to the effectiveness of the vaccine against COVID-19 in older 
adults. The fact that influenza vaccines only protect $56 \%$ of older adult people is explained by age-related immunity (Weiskopf et al, 2009). This shows that even after being vaccinated the older adults are still at risk of the mortality of the COVID-19 virus. According to the Lasso algorithm, age was a risk factor for patients with a serious illness and the risk of death rose as the age of patients with a serious illness increased by 5 years (Chowdurya et al, 2020).

The most significant risk factors for COVID-19-related mortality have been described as advanced age and co-morbid chronic diseases. This is due to the immune system ability of older adults and those with chronic diseases. Furthermore, older adults and people with chronic diseases are at a higher risk of contracting the disease. In addition, people with chronic diseases have higher levels of health anxiety, depression, and anxiety (Özdin \& Özdin, 2021). And individuals with the lack of health support or resources for these individuals, the anxiety could multiply and become a vicious cycle.

\section{Anxiety and depression due to COVID-19}

There are many reasons for anxiety and depression that causes by internal or external stress, in addition, the COVID-19 pandemic itself is a major cause of anxiety and depression to many people all around the world. As the world is combating the virus through the implementation of social distancing, more individuals are facing anxiety and depression due to isolation and staying inactive indoor. During the COVID-19 pandemics, people suffer varying degrees of psychological distress. Individuals with health issues, such as those who are suffering from chronic diseases are constantly worrying about their health problems due to being high risks and thus limit them from leaving their home to seek medical support that they need to keep healthy. While social distancing provides a method for protection against COVID-19, it also limits individuals' ability to seek attention and support from their family and their loved ones. Individuals with financial distress or who are laid off from work due to the economy finds it difficult to work and the stress of not able to finance themselves while being in lockdown thus causes stress. Parents and children in lockdown are not able to lead a normal social life with friends and extended family for an extended period also results in anxiety and depression. Many individuals who need emotional support from supportive groups are now in isolation are experiencing more psychological distress and especially those who are hospitalized due to COVID-19 as well as their family members and those whose family members died from COVID-19. In pandemics, a low level of health anxiety can lead to circumstances that negatively impact people's lives from a variety of angles due to a failure to follow preventive measures (Özdin \& Özdin, 2021). The fear for one's life is also an additional factor to stress and anxiety that could lead to depression. Although alternate theories cannot be ruled out, the findings of many studies indicate that the COVID-19 pandemic harms the psychological well-being of the general world population (Sønderskov et al, 2020).

\section{Effect of anxiety and depression on the immune system}

It's been 20 years since the first symptoms of immune disruptions in depression were identified (Hou \& Baldwin, 2012). In the state of anxiety and depression, an individual tends to ignore basic needs. Different individuals respond to depression differently. Some individuals ignore basic self-care and hygiene, whereas some tend to either lose sleep or 
excessive sleep while some develop an eating disorder such as overeating or skipping meals. Excessive worrying interferes with everyday functioning and lowers the quality of life in most people. (Hou \& Baldwin, 2012) Furthermore, it causes deeper anxiety and depression which is a vicious cycle to break especially during the pandemic when external support is not available due to social distancing.

Do anxiety and depression affect the immune system or does the disruptive immune system affect anxiety and depression? Depression may be caused by acquired and or innate immune responses to both external and internal stressors (Hou \& Baldwin, 2012). Many researchers have spent decades attempting to determine whether psycho-social causes, such as stress or depression, are linked to the occurrence, progression, or outcome of physical illness. There is substantial evidence that both traumatic life events and depressive conditions are linked to higher morbidity and mortality. In recent years, a significant body of research has looked into the possibility that immune system changes are linked to depressive disorders and depressive symptoms correlated with stressful life events. The associated immunologic changes have been linked to an increased risk of immune-related disease states such as cancer, autoimmune disorders, and infections, including the human immunodeficiency virus such as HIV (Stein et al, 1991). Thus this goes to support the opinion that individuals with anxiety and depression may be caused the individuals to be more at high risk of COVID-19 infections before the individuals' are being vaccinated.

Chronic anxiety has been shown in human studies to harm the immune system, resulting in impaired cellular and humoral immune responses as well as an increased occurrence of viral and bacterial infections (Hou \& Baldwin, 2012). Which are common scenarios seen in many cases. More obvious physical illnesses are in response to the bad habits developed due to anxiety and depression. For an instance, individuals who developed overeating habits due to anxiety and depression will face physical illness such as hypertension or obesity and thus makes the individuals even more at a higher risk of diseases. Anxiety is also linked to a weakened immune response to antiviral/bacterial vaccines, including those for hepatitis $B$, pneumococcal bacteria, rubella virus, meningitis virus, and influenza virus. (Hou \& Baldwin, 2012) This brings concern to the response of COVID-19 response in individuals suffering from anxiety and depression. The response to the COVID-19 vaccine on individuals with chronic depression is yet to be studied and evaluated to date, but this subject is important to be examined soon.

There's growing interest in the potential therapeutic benefit of treating depression by affecting the immune system, which has health implications. Given the facts, it is premature to speculate on the therapeutic impact of depression medication on immune competence and disease. Nonetheless, psychosocial therapies have a significant impact on the quality of under a light life and mental health of chronically ill people. Furthermore, a strong link between psycho-social states and immune status should not be needed to use these treatments in the treatment of patients with immune-related diseases such as cancer, AIDS, and autoimmune disorders (Stein et al, 1991). 


\section{Anxiety and Depression among the older adults due to COVID-19}

Most of the initial concern about how older adults would respond to COVID-19 stemmed from the fear that lockdown interventions would intensify depression and isolation. Loneliness has been shown to harm older adults (Vahia et al, 2020). The older adults living in homes or residential facilities depend highly on support from close family members or health professionals. Many older adults who are self-dependent life alone in isolation and occasionally being visited by close family and friends. The social distancing due to COVD-19 however will make isolation even stressful for them. This was a problem for older adults at home and in residential care facilities, where interaction with friends, relatives, and caregivers had become minimal (Vahia et al, 2020).

Many older adults lack the tools necessary to cope with COVID-19's stress. This may include material such as a lack of access and knowledge to smart technology for essentials such as food and goods delivery, social interaction such as the lack of attention and care from family and friends, or cognitive or biological needs such as the inability to engage in physical activity or participate in tasks or routines (Vahia et al, 2020). One of the effects of anxiety on depression by older adults during the COVID-19 pandemic variable is for basic needs such as wellbeing, treatment, cleaning, and food to increase due to the pandemic. Some needed to lift the bans of lockdown such as going out, socializing, walking, and exercising outdoor. Some older adults need permission to meet their children and other family members and that the lockdown bans needed to be lifted. The need for psychological support such as therapy, respect, love, tolerance, and care. The highest effect of anxiety on depression among older adults is the need for permission to meet their children and other family members and the lowest effect of anxiety on depression is among those who want the lockdown bans needed to be lifted.

Experts draw attention to the older adults among populations who may need special intervention. The older adults, who are a fragile group, may not find the tolerance they expect in this process. This can create a sense of worthlessness in older adults, leading them to frustration, depression, and anxiety. Living alone, loneliness and social isolation are wellknown risk factors for psychological disorders such as suicide, depression, and anxiety in advanced life (Wand et al, 2020). In this study, the highest and lowest effects of anxiety on depression were observed among the single and married older adults respectively. The older adults who had to stay at home during the pandemic who live with their spouses or have access to resources of social support from their children even if their spouse is dead might have lower anxiety levels and lower susceptibility to depression than those of the single older adults (Yildirim et al, 2020).

\section{Conclusion}

The great pandemic of 2020 has been unique stress that has affected communities all around the world. However, several individual reports from various countries have shown that at least some older adults are not experiencing harmful mental health effects commensurate with the elevated threats they faced during the early months of the COVID-19 pandemic. Understanding the causes and pathways that contribute to this resilience will help direct 
prevention strategies for other elderly people and groups whose mental wellbeing is more seriously impaired. Such approaches such as increasing components of wisdom like emotional regulation, empathy, and compassion.10 It would also be useful to consider how technology may be leveraged to this end. However, it is critical to recognize that these positive early findings notwithstanding, careful monitoring and additional research will be needed to understand the psychological and mental health effects of the ongoing COVID-19 pandemic among the older population (Vahia et al, 2020).

Various measures such as social distancing, quarantine, and self-isolation are taken to reduce the spread of the virus during the pandemic. However, the number of people affected by the disease and death rates are increasingly rising (Xu et al., 2020). All these factors can have negative effects on the mental health of society (Yildirim et al, 2020). The older adults and those with chronic disease are those who are very vulnerable to the pandemic and although vaccination is now available the effectiveness of the vaccine is yet to be determined as the sole prevention of the COVID-19 disease.

\section{CONFLICTS OF INTEREST}

There are no financial issues or interest of conflicts to declare.

\section{REFERENCES}

[1] Barton, F., Haynes, B. F., Corey, L., and Fernandes, P. (2020). Prospects for a safe COVID-19 vaccine. Science Translation Medicine, 12, eabe0948.

[2] Chen, Y., Klein, S. L., Garibaldi, B. T., et al. (2021) Aging in COVID-19: Vulnerability, immunity and intervention. Ageing Research Reviews, 65, 101205.

[3] Guo, Y.-R., Cao, Q.-D., Hong, Z.-S., Tan, Y.-Y., Chen, S.-D., Jin, H.-J., et al. (2020) The origin, transmission and clinical therapies on coronavirus disease 2019 (COVID-19) outbreak - an update on the status. Military Medical Research.

[4] Hou, R., and Baldwin, D. S., (2012) A neuroimmunological perspective on anxiety disorders. Human Psychopharmacology, 27, 6-14.

[5] Hyland, P., Shevlin, M., and McBride, O. (2020) Anxiety and depression in the Republic of Ireland during the COVID-19 pandemic. Acta Psychiatr Scand, 142, 249256.

[6] Leonard, B. E. (2010) The concept of depression as a dysfunction of the immune system. Curr Immunol, 6(3), 205-212. doi:10.2174/157339510791823835.

[7] Karasar, B., and Canl, D. (2020) Psychological resilience and depression during the covid-19 pandemic in turkey. Psyiachitra Danubina, 32(2), 273-279.

[8] Nascimento, I. J. B. D., Cacic, N., Abdulazeem, H. M., Groote, T. C. V., et al. (2020) Novel Coronavirus Infection (COVID-19) in Humans: A Scoping Review and Meta-Analysis, Journal of Clinical Medicine, 9:941. doi:10.3390/jcm9040941. 
[9] Prompetchara, E., Ketloy, C., and Palaga, T. (2020) Immune responses in COVID-19 and potential vaccines: lessons learned from SARS and MERS epidemic. Asian Pac J Allergy Immunol.

[10] Sønderskov, K. M., Dinesen, P. T., Santini, Z. I., and Østergaard, S. D. (2020) Thedepressive state of Denmark during the COVID-19 pandemic. Acta Neuropsychiatrica, 32, 226-228. doi: 10.1017/neu.2020.15

[11] Stein, M., Miller, A. H., and Trestman, R. L., (1991) Depression, the Immune System, and Health and Illness. Archive of General Psychiatry, 48, 171-177.

[12] Vahia, I. V., Jeste, D. V., and Reynolds III, C. F., (2020) Older adults and the mental health effects of Covid-19. American Medical Association, 324(22), 2253-2254.

[13] Wand, A. P. F., Zhong, B.-L., Chiu, H. F. K., Draper, B., \& De Leo, D. (2020). Covid-19: The implications for suicide in older adults. International Psychogeriatrics, $\quad$ 32(116), 1225-1230.

[14] Weiskopf, D., Weinberger, B., and Grubek-Loebenstein, B., (2009) The aging of the nimmune system. European Society for Organ Transplantation, 22, 1041-1050.

[15] World Health Organization (2020) Coronavirus disease (COVID-19) Pandemic. Available at https://www.who.int/emergencies/diseases/novelcoronavirus-2019.

[16] Xu, X.-W., Wu, -X.-X., Jiang, X.-G., Xu, K.-J., Ying, L.-J., Ma, C.-L., and Gao, H.-N. (2020). Clinical findings in a group of patients infected with the 2019 novel coronavirus (SARSCov-2) outside of Wuhan, China: Retrospective case series. $\quad$ bmj, 368, 1-7.

[17] Yildirim, H., Isik, K., and Aylaz, R., (2020) The effect of anxiety levels of older adults people in quarantine on depression during COVID-19 pandemic. Social Work in Public Health.

[18] Zhang, Y., \& Ma, Z. F. (2020). Impact of the COVID-19 pandemic on mental health and quality of life among local residents in Liaoning Province, China: A cross-sectional study. International Journal of Environmental Research and Public Health, 17(7), Article 2381. https://doi.org/10.3390/ijerph17072381. 